ARTICLE

Received 17 Mar 2013 | Accepted 17 Jun 2013 | Published 12 Jul 2013

DOI: $10.1038 /$ ncomms3162

OPEN

\title{
Conducting linear chains of sulphur inside carbon nanotubes
}

Toshihiko Fujimori ${ }^{1}$, Aarón Morelos-Gómez ${ }^{2}$, Zhen Zhu ${ }^{3}$, Hiroyuki Muramatsu ${ }^{4}$, Ryusuke Futamura1, Koki Urita ${ }^{5}$, Mauricio Terrones ${ }^{1,6}$, Takuya Hayashi ${ }^{7}$, Morinobu Endo ${ }^{1}$, Sang Young Hong ${ }^{8}$, Young Chul Choi ${ }^{8}$, David Tománek ${ }^{3}$ $\&$ Katsumi Kaneko ${ }^{1}$

Despite extensive research for more than 200 years, the experimental isolation of monatomic sulphur chains, which are believed to exhibit a conducting character, has eluded scientists. Here we report the synthesis of a previously unobserved composite material of elemental sulphur, consisting of monatomic chains stabilized in the constraining volume of a carbon nanotube. This one-dimensional phase is confirmed by high-resolution transmission electron microscopy and synchrotron X-ray diffraction. Interestingly, these one-dimensional sulphur chains exhibit long domain sizes of up to $160 \mathrm{~nm}$ and high thermal stability ( $~ 800 \mathrm{~K})$. Synchrotron X-ray diffraction shows a sharp structural transition of the one-dimensional sulphur occurring at $\sim 450-650 \mathrm{~K}$. Our observations, and corresponding electronic structure and quantum transport calculations, indicate the conducting character of the one-dimensional sulphur chains under ambient pressure. This is in stark contrast to bulk sulphur that needs ultrahigh pressures exceeding $\sim 90 \mathrm{GPa}$ to become metallic.

\footnotetext{
${ }^{1}$ Research Center for Exotic Nanocarbons (JST), Shinshu University, 4-17-1 Wakasato, Nagano 380-8553, Japan. ${ }^{2}$ Institute of Carbon Science and Technology, Shinshu University, 4-17-1 Wakasato, Nagano 380-8553, Japan. ${ }^{3}$ Department of Physics and Astronomy, Michigan State University, East Lansing, Michigan 48824, USA. ${ }^{4}$ Department of Materials Science and Technology, Nagaoka University of Technology, 1603-1, Kamitomioka, Nagaoka 9402188, Japan. ${ }^{5}$ Department of Applied Chemistry, Faculty of Engineering, Nagasaki University, 1-14 Bunkyo-machi, Nagasaki-shi, Nagasaki 852-8521, Japan. ${ }^{6}$ Department of Physics, Department of Chemistry, Department of Material Science and Engineering and Center for 2-Dimensional and Layered Materials, The Pennsylvania State University, University Park, Pennsylvania 16802, USA. ${ }^{7}$ Faculty of Engineering, Shinshu University, 4-17-1 Wakasato, Nagano 3808553, Japan. ${ }^{8}$ CNT Team, Hanwha Chemical Corporation, 80 Annamro 402 gil, Bupyeong-gu, Incheon 403-030, Republic of Korea. Correspondence and requests for materials should be addressed to T.F. (email: t_fujimori@shinshu-u.ac.jp).
} 
lemental sulphur, the most polymorphic element, is an - insulator in the bulk three-dimensional (3D) structure under ambient conditions. Only at ultrahigh pressures exceeding $\sim 90 \mathrm{GPa}$, does it undergo a structural phase transition to a base-centred orthorhombic metallic phase ${ }^{1-4}$. From this base-centred orthorhombic phase, it is possible to hypothetically extract a one-dimensional (1D) sulphur chain exhibiting a zigzag configuration. This $1 \mathrm{D}$ system has been predicted to show a finite electronic density of states near the Fermi level and thus to display metallic behaviour ${ }^{5-7}$. Furthermore, Springborg and Jones $^{5,6}$ have proposed that the metallic behaviour of sulphur could also result from a free-standing 1D linear configuration. Despite the fact that $3 \mathrm{D}$ sulphur behaves as an insulator ${ }^{1}$, to the best of our knowledge, the experimental isolation of 1D sulphur with metallic properties has not been reported so far, because it is almost impossible to preserve $1 \mathrm{D}$ chains without any supports.

A common problem found in free-standing $1 \mathrm{D}$ metallic systems is the Peierls instability associated with bond length alternation, which opens a fundamental energy gap and leads to a metal-insulator transition ${ }^{8}$ : $1 \mathrm{D}$ conducting polymers (for example, polyacetylene) constitute a well-known 1D system undergoing Peierls transition ${ }^{9}$. A different way to suppress the Peierls transition in 1D metallic systems that form zigzag or other nonlinear chains is to sterically constrain the nonlinear structure inside a narrow cylindrical cavity. Even small lattice distortions of the enclosed 1D metal may change the electronic structure near the Fermi level ${ }^{10}$ sufficiently to suppress the Peierls instability and preserve the enclosed nanowire as a $1 \mathrm{D}$ quantum conductor.

In this context, single-walled carbon nanotubes (SWCNTs) turn out to be superior host materials in comparison with other porous materials because of the presence of closed quasi-1D channels with diameter of $\approx 1 \mathrm{~nm}$ (refs 11-19). In addition, SWCNTs exhibit unique $1 \mathrm{D}$ electronic behaviour ranging from metallic to semiconducting, depending on the nanotube chirality (given by the diameter and the orientation of the hexagonal honeycomb along the tubule axis) ${ }^{20}$. Furthermore, carbon nanotubes (CNTs) are highly rigid because of the stability of $\mathrm{sp}^{2}$ carbon bonds. This rigidity efficiently suppresses the Peierls instability in metallic SWCNTs (M-SWCNT) ${ }^{21}$. The steric constraints provided by the enclosing rigid SWCNT should therefore also suppress a potential Peierls instability in the enclosed $1 \mathrm{D}$ chain.

Here we report the successful synthesis of 1D chains of sulphur inside SWCNTs and double-walled CNTs (DWCNTs) using a vapour reaction method. The modified protocol resembles the well-established procedure used to encapsulate fullerenes inside SWCNTs $^{22}$. We demonstrate the highly ordered 1D zigzag and linear sulphur chains with long domain sizes up to $160 \mathrm{~nm}$ and high thermal stability $(\sim 800 \mathrm{~K})$. Most importantly, the $1 \mathrm{D}$ sulphur chains exhibit a conducting character under ambient pressure, which is also supported by ab initio density functional calculations.

\section{Results}

Structural characterization of $1 \mathrm{D}$ sulphur chains. Figure 1a displays high-resolution transmission electron microscopy (HRTEM) images of sulphur encapsulated inside a SWCNT (S@SWCNT). In these images, we can identify two lines separated by $0.32 \mathrm{~nm}$ inside the hollow core of a SWCNT with diameter of $1.1 \mathrm{~nm}$, which we interpret as two parallel monatomic sulphur chains. Furthermore, those sulphur chains seem to move simultaneously along the tube axis, activated by electron-beam irradiation during the HRTEM observations (see Supplementary Fig. S1). Occasionally, the motion of the sulphur chains inside a SWCNT generated an arrangement with one chain obscuring the other in the HRTEM image. The surrounding CNT walls allowed us to identify the encapsulated structure with atomic resolution by stabilizing it in place and also by protecting it from electronbeam damage during imaging. For this reason, a clear 1D zigzag atomic chain of sulphur could be identified by HRTEM inside a DWCNT (S@DWCNT) with an inner diameter of $0.7 \mathrm{~nm}$, as depicted in Fig. 1b. A different linear sulphur chain was also observed inside a DWNT with a narrower inner diameter of $0.6 \mathrm{~nm}$ (Fig. 1c). Our HRTEM analysis reveals that the mean periodic distance in axial direction is $0.33 \pm 0.03 \mathrm{~nm}$ for the zigzag chains and $0.18 \pm 0.02 \mathrm{~nm}$ for the linear sulphur chains. From a theoretical standpoint, as the number of sulphur atoms per primitive unit cell is reduced from two to one during the zigzag-to-linear transition, we should compare supercells with two sulphur atoms, independent of conformation. In this case, the periodic distance of the two-atom supercell of the linear chain is $0.36 \pm 0.04 \mathrm{~nm}$, larger than that of the zigzag chain. This agrees with our expectation that with decreasing nanotube diameter, the constrained sulphur chains generally become straighter, and their periodic distance in axial direction increases (see Supplementary Fig. S2).

Our X-ray diffraction (XRD) analysis reveals the presence of highly ordered zigzag or linear atomic sulphur chains (Fig. 1d). The XRD pattern of S@SWCNTs clearly shows asymmetric
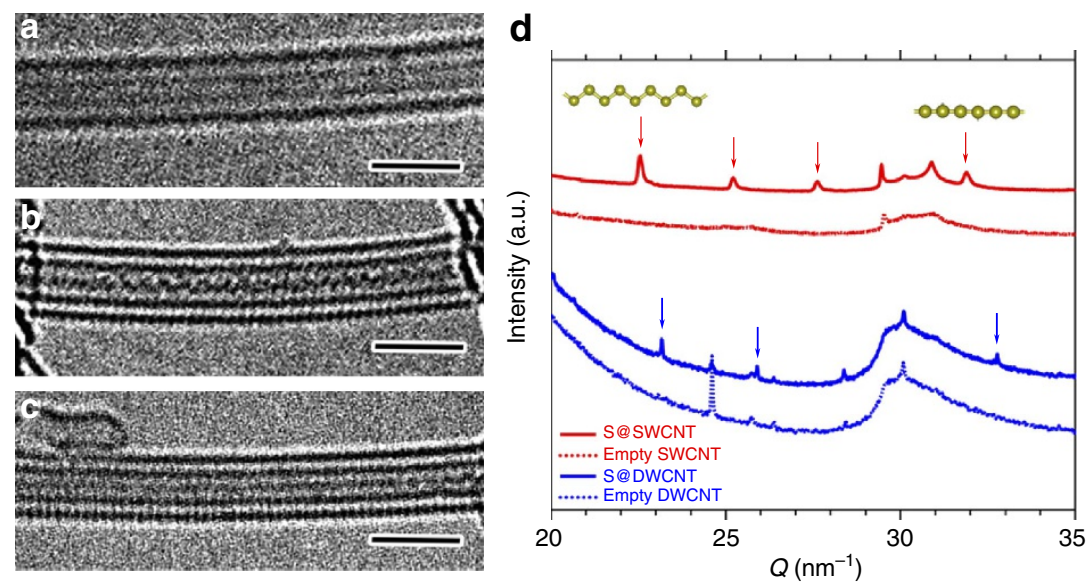

Figure 1 | Structural identification of 1D sulphur chains inside CNTs. (a) HRTEM image of S@SWCNT. The two lines correspond to 1D sulphur chains encapsulated inside a SWCNT. (b) HRTEM image of S@DWCNT with the 1D sulphur chain in zigzag conformation. (c) A 1D linear chain inside a DWCNT. Scale bar, 2 nm. (d) XRD profiles of S@SWCNTs, empty SWCNTs, S@DWCNTs and empty DWCNTs. Arrows indicate the Bragg peaks of $1 \mathrm{D}$ sulphur chains. 
lambda-shaped Bragg peaks at the scattering vectors $Q=22.5$, 25.2, 27.6 and $31.9 \mathrm{~nm}^{-1}$, which are characteristic of a 1D structure ${ }^{14,23-25}$. These $Q$-values correspond to $1 \mathrm{D}$ lattice constants $d$ ranging from $d=0.197$ to $0.279 \mathrm{~nm}$. This particular distribution of lattice constants reflects the different degrees of distortion, to which the enclosed sulphur chains are subjected to by their surrounding nanotubes with a particular diameter distribution. Different lattice constants may occur in a single sulphur chain inside a defective CNT that may change its diameter or pin down sulphur atoms at wall defects. The shortest $0.197 \mathrm{~nm}$ spacing is only a little shorter than the bond length in other sulphur allotropes (for example, $0.2046 \mathrm{~nm}$ in $\alpha-S_{8}$ ring molecule, $0.2066 \mathrm{~nm}$ in fibrous sulphur $\left.S_{\psi}\right)^{1}$. We thus associate $d=0.197 \mathrm{~nm}$ with a linear chain and $d=0.279 \mathrm{~nm}$ with a zigzag chain encapsulated inside a SWCNT. The 1D sulphur chains inside DWCNTs also exhibit zigzag and linear conformations, as observed in HRTEM images shown in Fig. 1b,c. The axial lattice constant values ranging from $d=0.192$ to $0.271 \mathrm{~nm}$ were slightly shorter than inside SWCNTs. Also, these $d$ values show good agreement with the HRTEM analysis, which reveals the mean periodic distances to be $0.18 \pm 0.02 \mathrm{~nm}$ in linear and $0.33 \pm 0.03 \mathrm{~nm}$ in zigzag sulphur chains (see Supplementary Fig. S2). Therefore, the 1D sulphur chains encapsulated inside SWCNTs and DWCNTs are very similar, except for a slight axial compression inside the DWCNTs.

The domain size of the 1D chains of sulphur, $\xi$, has a crucial role in electric transport properties, because ends of short sulphur chain segments act as scattering centres and alter electron transport of the entire system. The domain size is estimated using $\xi=2 \pi / \Delta Q$, where $\Delta Q$ is the full width at half maximum of the corresponding Bragg peak ${ }^{14}$. In particular, the domain size of the 1D sulphur chains inside SWCNTs is $35-45 \mathrm{~nm}$, and that within DWCNTs is $90-160 \mathrm{~nm}$ at $300 \mathrm{~K}$. Assuming that the typical length of a CNT is $\sim 1 \mu \mathrm{m}$, S@SWCNTs contain $\sim 20-30$ domains and S@DWCNTs contain 6-10 domains of 1D sulphur chains. We interpret this long-range order and large domain size as a consequence of the strong covalent bond character within the 1D sulphur chains.

We further tested the temperature dependence of the 1D Bragg peaks to assess the thermal stability and phase transitions of the 1D sulphur chains in the zigzag and linear geometries. For S@SWCNTs consisting of two parallel 1D chains of enclosed sulphur, the $Q$-values of Bragg peaks assigned to zigzag and linear lattices decrease slightly as the temperature increases up to $800 \mathrm{~K}$ (Fig. 2a). This thermal contraction, driven by configurational entropy, is rather common in polymers. Though $\Delta Q$ values become broad at temperatures higher than $650 \mathrm{~K}$, the $1 \mathrm{D}$ Bragg peaks are still pronounced at $800 \mathrm{~K}$, indicating a high thermal stability of the 1D double chains of sulphur. For DWCNTs encapsulating single 1D chains in the inner tube, we found an abrupt decrease in the $Q$-value of the peak at $T \approx 650 \mathrm{~K}$ (Fig. 2b), implying a large structural change in this $1 \mathrm{D}$ system. Interestingly, the lattice constants of the 1D chains in S@DWCNTs closely approach the values found in S@SWCNT >650 K (Fig. 2c,d). This result indicates that SWCNTs constitute the most suitable cavities for stabilizing $1 \mathrm{D}$ chains of sulphur. In comparison with bulk arrays of $\mathrm{S}_{8}$ ring molecules (the most stable sulphur allotrope with melting point at $\sim 393 \mathrm{~K}$ and boiling point
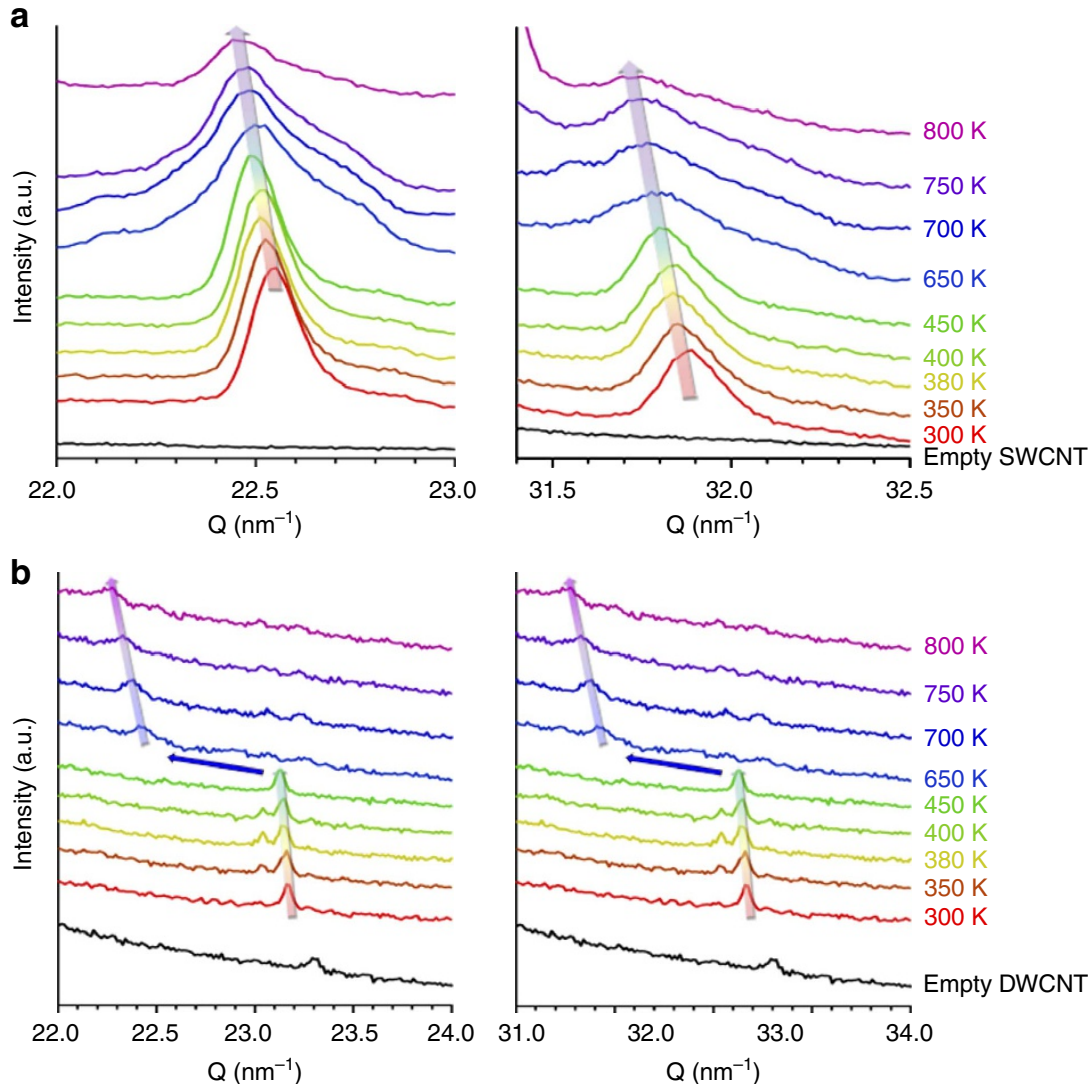
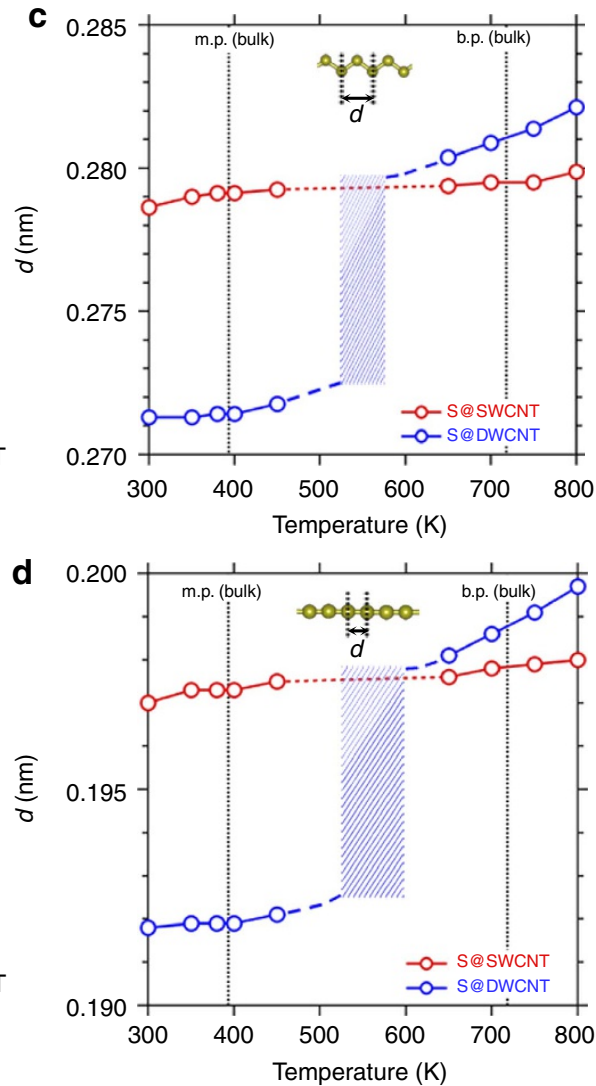

Figure 2 | Thermal stability of 1D sulphur chains with zigzag and linear geometries. Temperature dependence of the 1D Bragg peaks of sulphur inside (a) SWCNTs and (b) DWCNTs. Temperature dependence of the axial lattice constant $d$ in (c) 1D zigzag and (d) linear sulphur chains. The melting point (m.p.) and boiling point (b.p.) of bulk sulphur $\left(\alpha-S_{8}\right)$ are shown for comparison. A sharp increase in the $d$ value of S@DWCNT between 450-650 K indicates a structural change of the 1D chains. 
at $~ 718 \mathrm{~K}), 1 \mathrm{D}$ sulphur chains in both S@SWCNT and S@DWCNT display very different thermodynamic behaviour. Very different from the bulk sulphur phase, which undergoes complicated crystallographic and structural transitions 1,26 including 3D trigonal and tetragonal chains at high pressures ${ }^{27-29}$, sulphur chains are immobilized in the host CNTs up to $800 \mathrm{~K}$.

To understand the chemical bonding of 1D sulphur chains, we performed X-ray photoelectron spectroscopy (XPS) studies in the S2s and S2p core-level regions (Fig. 3a,b) and resonance Raman spectroscopy in the frequency range where the characteristic conformation of S-S bonding could be extracted for the 3D bulk systems (see Supplementary Fig. S3). The XPS S $2 s$ and S2p spectra of S@SWCNTs and S@DWCNTs look very similar, indicating that the chemical bonding of sulphur atoms in the chains is identical, as already suggested by the crystallographic information extracted from the XRD analysis. As seen in Fig. 3a,b, the S2s and S2p core-level binding energies of the $1 \mathrm{D}$ sulphur chains are smaller than those of the bulk $S_{8}$ ring molecule (see Supplementary Table S1, Supplementary Fig. S4 and Supplementary Note 1 for details). The lower binding energies reflect the strain, to which sulphur chains enclosed inside CNTs are subjected. Unlike the bulk $3 \mathrm{D}$ sulphur ${ }^{30}$, the $\mathrm{S}-\mathrm{S}$ stretching modes are obscured in resonance Raman spectra of the strained sulphur chains stabilized inside CNTs (see Supplementary Fig. S3), implying that the encapsulated sulphur chains exhibit a 1D planar conformation. It should also be noted that the XPS C1s core-level spectra of the enclosing CNTs (see Supplementary Fig. S5 and Supplementary Note 2) are nearly unaffected by the presence of enclosed sulphur chains. This indicates that there is no or only a negligible chemical interaction between the sulphur chains and the host CNTs. The absence of C1s core-level shifts due to the presence of sulphur also indicates that the charge transfer between the nanotubes and sulphur chains should be negligible. This agrees with the computational results presented below and can be rationalized by the fact that the electronegativity of sulphur and carbon has the same value of 2.5 on the Pauling scale 31 .

The intensity analysis of our XPS spectra indicates that an atomic filling ratio of sulphur is 6.7 atom $\%(16 \mathrm{wt} \%)$ for S@SWCNT (Fig. 3a). This is in good agreement with the thermogravimetric (TG) analysis obtained during combustion, indicating $12 \mathrm{wt} \%$ of filled sulphur, as seen in Fig. 3c. Our XPS analysis of S@DWCNT indicates a lower S filling ratio of 3.5 atom\% $(8.8 \mathrm{wt} \%)$. This quantity could not be verified by TG analysis, because the combustion temperature of DWCNTs and sulphur is the same (Fig. 3d). These high atomic filling ratios, together with the results of our elemental mapping analysis that find coinciding spatial distribution of carbon and sulphur (see Supplementary Figs S6 and S7 and Supplementary Note 3), indicate that sulphur is uniformly distributed inside the CNTs.

Conducting character of 1D sulphur chains. Raman spectroscopy not only probes vibration spectra but also can be used to extract information about metallicity of the 1D sulphur chains. Specifically, an asymmetric Raman signal in the low-frequency shoulder of the tangential $\mathrm{G}^{+}$-mode at $\sim 1590 \mathrm{~cm}^{-1}$, the socalled Breit-Wigner-Fano (BWF) line, is associated with metallic
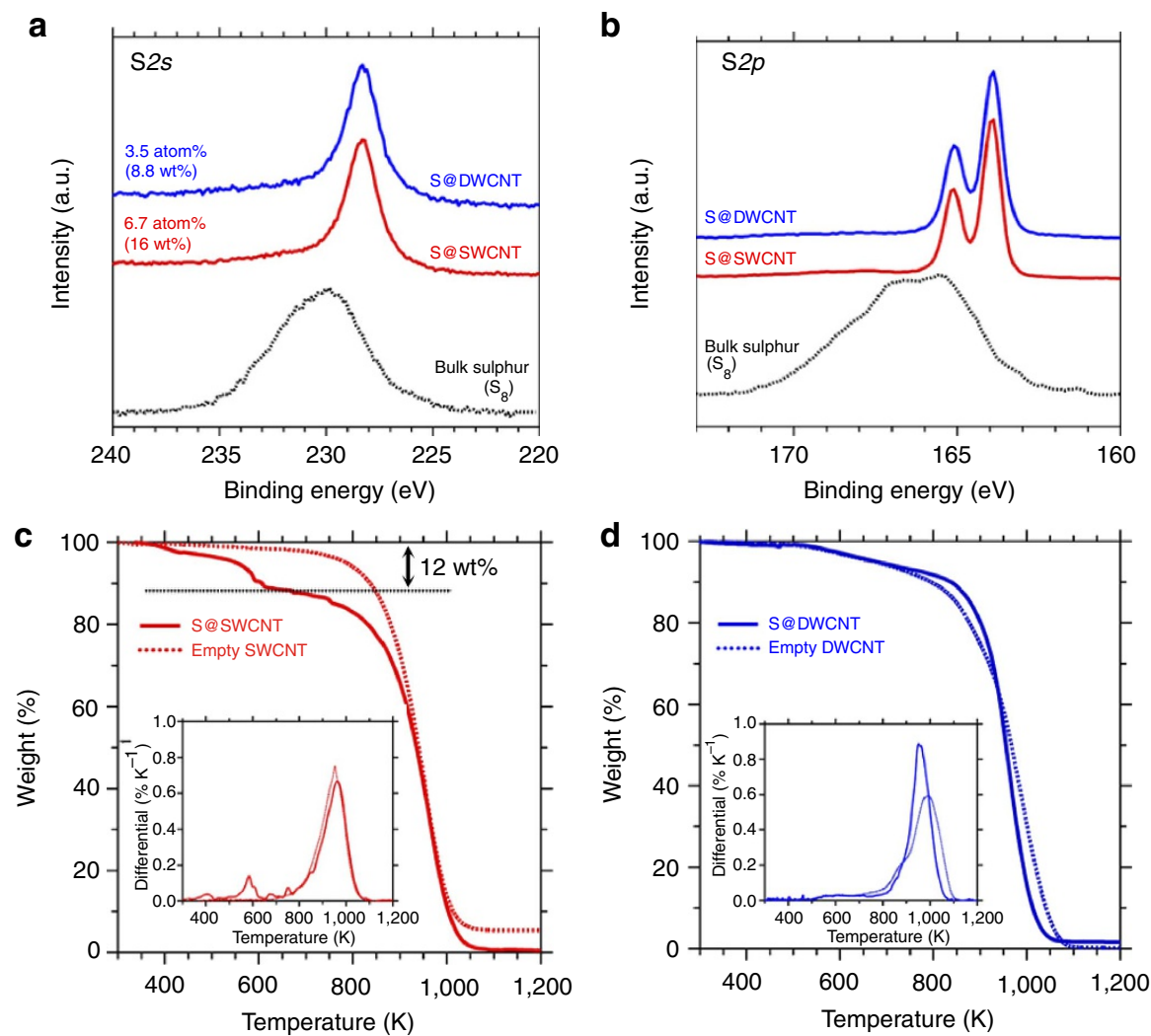

Figure 3 | Chemical states and filling ratios of 1D sulphur chains. XPS spectra in the (a) S2s and (b) S2p core-level regions of empty SWCNTs, S@SWCNTs, empty DWCNTs and S@DWCNTs, in comparison with bulk sulphur $\left(\alpha-S_{8}\right)$. Filling ratios of the 1D sulphur chains, evaluated by XPS analysis, are 6.7 atom\% (16 wt\%) for S@SWCNTs and 3.5 atom\% (8.8 wt\%) for S@DWCNTs. TG curves of (c) empty SWCNTs and S@SWCNTs and (d) empty DWCNTs and S@DWCNTs, measured under $\mathrm{He} / \mathrm{O}_{2}$ gas flow ( $\mathrm{He}: \mathrm{O}_{2}=8: 2$ ). Insets show the differential TG curves. TG analysis reveals that the filling ratio is 12 wt\% for S@SWCNTs, in good agreement with values obtained by XPS. As the oxidation reactions start at nearly the same temperature for DWCNTs and 1D chains of sulphur encapsulated in DWCNTs ( $\sim 800 \mathrm{~K})$, the filling ratio of S@DWCNTs could not be evaluated by TG analysis. 
character in graphitic materials including $\mathrm{M}-\mathrm{SWCNTs}^{32-35}$. Figure 4 compares the Raman spectra of S@SWCNTs and S@DWCNTs with those of empty CNTs. We chose two different excitation wavelengths, $\lambda=532$ and $785 \mathrm{~nm}$, to selectively probe either semiconducting or metallic CNTs under resonant Raman conditions, which depend on the electronic structure of the corresponding $\mathrm{CNTs}^{36}$. Among our SWCNT samples, M-SWCNTs represent the main component in the spectrum obtained using the $785 \mathrm{~nm}$ laser excitation, whereas semiconducting SWCNTs dominate the $532 \mathrm{~nm}$ laser excitation spectrum (see Supplementary Figs S8 and S9). The BWF line turns out to be more intense in the presence of encapsulated 1D sulphur chains within M-SWCNTs (Fig. 4a), thus implying that the presence of sulphur enhances the electronic conduction of CNTs. This result provides evidence that sulphur chains constrained in the nanotube enclosure are metallic (see Supplementary Note 4), thus enhancing the electrical conductivity of the carbon-sulphur hybrid system.

To learn more about specific conditions of sulphur-related conductivity enhancement, we compared the BWF lines in Raman spectra of metallic and semiconducting CNTs enclosing sulphur chains in Fig. 4. Unlike sulphur in a M-SWCNT shown in Fig. 4a, no BWF resonance occurs because of the presence of sulphur in a semiconducting SWCNT (Fig. 4b). Moreover, in a DWCNT with a metallic outer tube in resonance with the $785 \mathrm{~nm}$ laser excitation (see Supplementary Fig. S10) and a semiconducting inner tube, the presence of sulphur does not enhance the intensity of the BWF line in the outer wall (Fig. 4c). For a DWCNT with a semiconducting outer tube and a metallic inner tube in resonance with the $532 \mathrm{~nm}$ laser excitation (see Supplementary Fig. S11), the BWF line is slightly enhanced in the presence of encapsulated 1D sulphur chains (Fig. 4d). These results suggest a direct contact between the metallic 1D sulphur chains and the metallic character of the enclosing CNTs, which seems to be the key for enhancing the electrical conductivity of hybrid carbon-sulphur systems.

Not only the Raman-active $\mathrm{G}^{+}$-mode but also the $2 \mathrm{D}$ mode (see Supplementary Table S2) reflect the interaction of enclosed sulphur chains with the enclosing wall in S@SWCNTs. We concluded that the presence of enclosed sulphur does not change the $\mathrm{G}^{+}$-band frequency, indicating the absence of strong chemical bonds or charge transfer in the system (see Supplementary Note 5). The absence of charge transfer had been confirmed independently by XPS spectra (see Supplementary Fig. S5). In contrast to the $\mathrm{G}^{+}$-mode, the $2 \mathrm{D}$ blue band shifts up to $9 \mathrm{~cm}^{-1}$ due to enclosed sulphur chains. Although such frequency shifts of the $2 \mathrm{D}$ mode may be caused by chemical doping of nanotubes ${ }^{37}$, the blue shift in this case is caused by the presence of the sulphur chain inside the nanotube which hinders the motion of carbon atoms and thus hardens the vibrational modes.

In order to verify that the presence of $1 \mathrm{D}$ sulphur chains indeed enhances the electron transport in nanotubes containing sulphur chains, we performed direct-current electrical resistance measurements for buckypapers containing empty and sulphurfilled metallic CNTs, as well as pristine M-SWCNTs, and report our findings in Fig. 5a. Our results indicate a significant drop in resistivity caused by encapsulation of $1 \mathrm{D}$ sulphur chains in
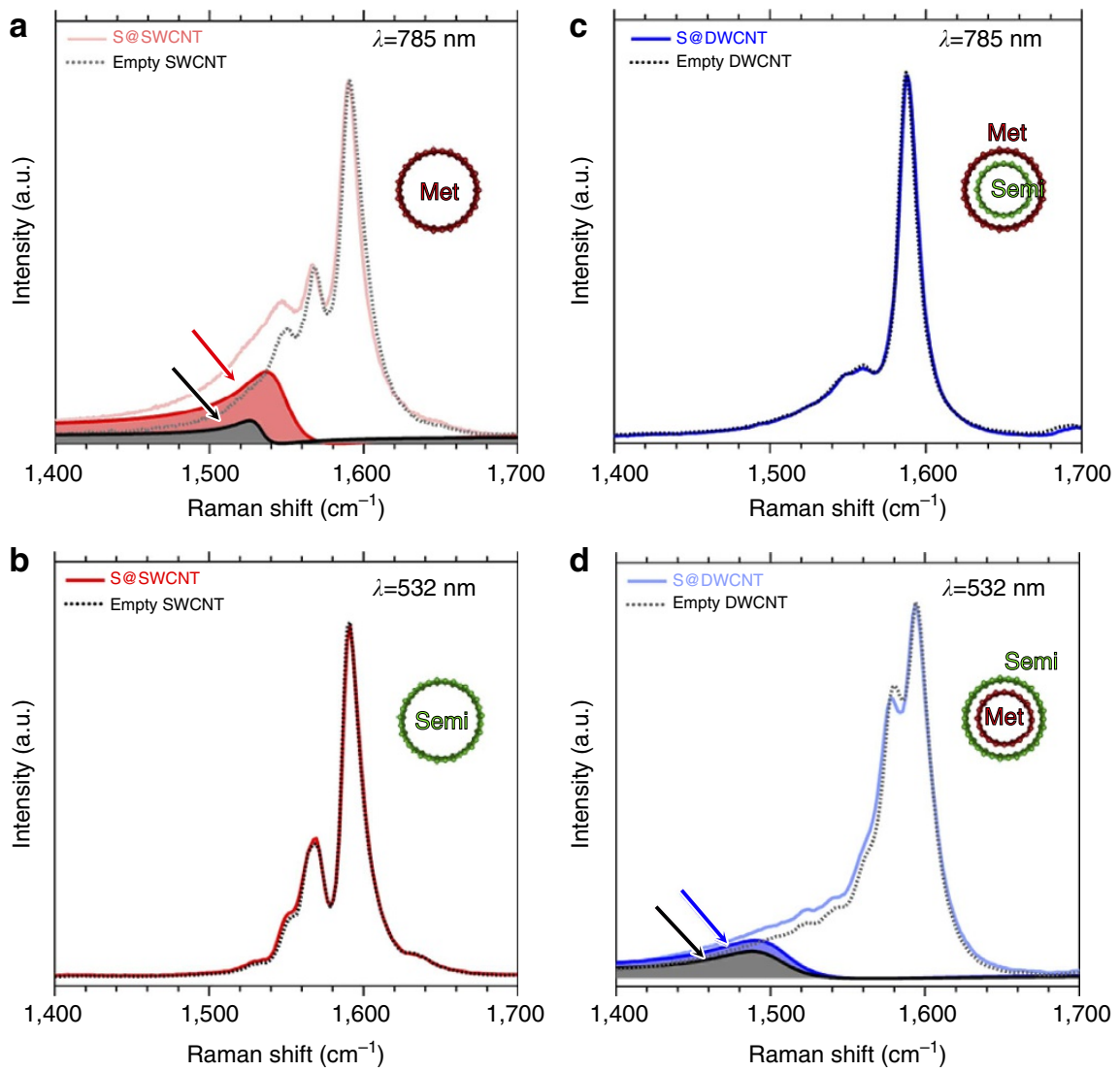

Figure 4 | Spectroscopic evidence for metallicity of 1D sulphur chains. Resonance Raman spectra of S@SWCNTs in comparison with empty SWCNTs, measured with $785 \mathrm{~nm}$ (a) and $532 \mathrm{~nm}$ (b) laser excitations. Raman spectra of empty DWCNTs and S@DWCNTs, measured with $785 \mathrm{~nm}$ (c) and $532 \mathrm{~nm}$ (d) laser excitations. The asymmetric BWF lines associated with metallic CNTs or enhanced in presence of metallic 1D sulphur chains are highlighted by the arrows in a and d. Met, metallic CNT; Semi, semiconducting CNT. 

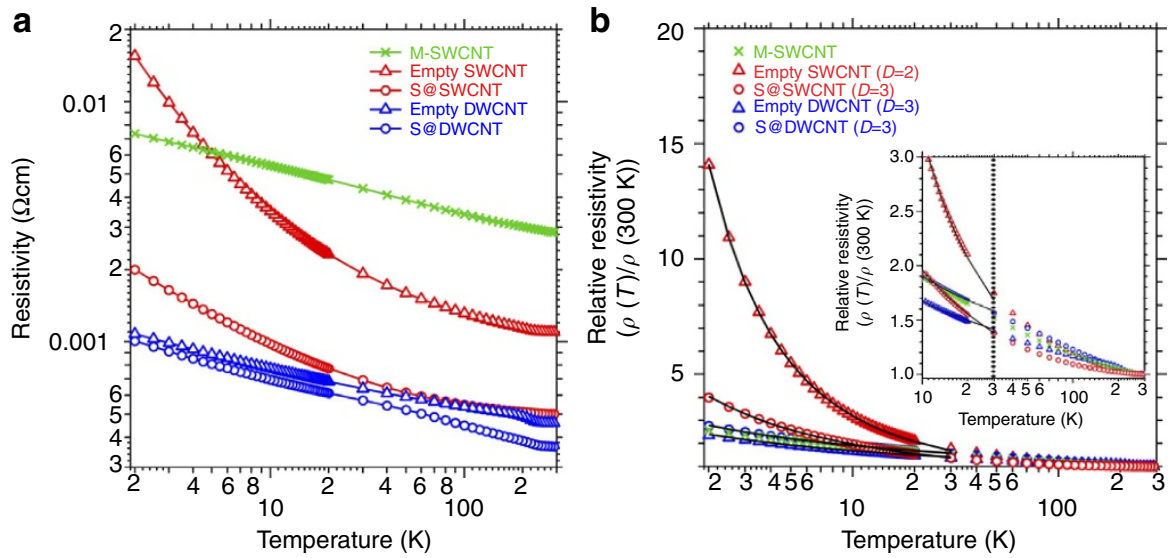

Figure 5 | Electric transport of buckypapers containing 1D sulphur chains. (a) Resistivity of films (buckypapers) consisting of empty SWCNTs, S@SWCNTs, empty DWCNTs and S@DWCNTs as a function of temperature, in comparison with pristine M-SWCNTs. (b) Temperature dependence of the relative film resistivity, normalized by the $T=300 \mathrm{~K}$ value, fitted with the variable range hopping model (black solid lines). $D$ represents the dimensionality of electron hopping in these systems. The inset in $\mathbf{b}$ shows the magnified relative resistivity curves (10-300 K). The relative resistivity curve of M-SWCNTs could not be reproduced using the variable range hopping model ${ }^{41}$.

SWCNTs. The temperature-dependent measurements indicate that the resistivity of all systems decreases with increasing temperature, which is characteristic of systems with a low density of states at $E_{\mathrm{F}}$. At $300 \mathrm{~K}$, we observe that the electric resistivity $\rho(300 \mathrm{~K})$ of SWCNTs drops by one half from $1.1 \times 10^{-3}$ to $5.0 \times 10^{-4} \Omega \mathrm{cm}$. At $2 \mathrm{~K}$, the resistivity values for pristine SWCNTs corresponding to $\rho(2 \mathrm{~K})=1.5 \times 10^{-2} \Omega \mathrm{cm}$ drop by a whole order of magnitude to $2.0 \times 10^{-3} \Omega \mathrm{cm}$ because of the presence of enclosed sulphur chains. The same general trends, but even with lower resistivity values across the whole temperature range, occur in pristine and S-filled DWCNT systems when compared to the equivalent SWCNT systems. We need to point out that resistivity measurements in buckypapers are strongly affected by the morphology of the sample, which consist of aggregates of entangled CNT bundles of different length connected at many unspecific contact points. The observed low resistivity of M-SWCNTs in comparison with other empty CNTs, may be caused not only by their intrinsic metallic character but also by their shorter average length, a consequence of the dispersion/separation process ${ }^{38,39}$ that affects the buckypaper morphology. Assuming that the contact resistance within the buckypaper is temperature independent, we normalized the resistivity $\rho(T)$ at a given temperature by $\rho(300 \mathrm{~K})$ to suppress the effect of different contact morphologies and plotted the temperature-dependent relative resistivity $\rho(T) / \rho(300 \mathrm{~K})$ in Fig. 5b. As suggested earlier, also the relative resistivity of S@SWCNTs is lower than that of empty SWCNTs, indicating additional electron transport channels present in the hybrid system.

We modelled the observed conductance data by the variable range hopping model ${ }^{40-43}$ to evaluate the dimensionality of percolation paths due to electron hopping. We found the relative resistivity curve $\rho(T) / \rho(300 \mathrm{~K})$ of S@SWCNTs to be well reproduced by assuming $3 \mathrm{D}$ hopping processes, whereas that of empty SWCNTs follows a $2 \mathrm{D}$ hopping behaviour. The corresponding fits, shown by the black lines in Fig. 5b, indicate that the 1D sulphur chains establish new conduction paths throughout the system. We find the relative resistivity of S@SWCNT to be comparable to that of M-SWCNTs $>10 \mathrm{~K}$, displaying the lowest resistivity values among the samples.

$A b$ initio quantum conductance study of $1 D$ sulphur chains. To better understand the stability and electronic properties of these novel hybrid systems, we performed $a b$ initio density functional a
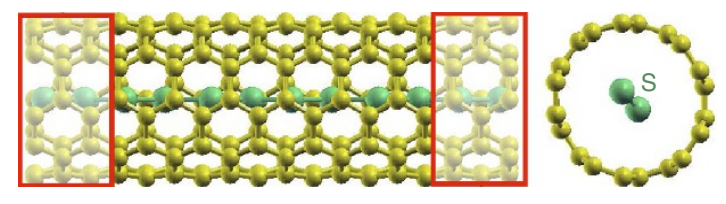

Lead

Lead
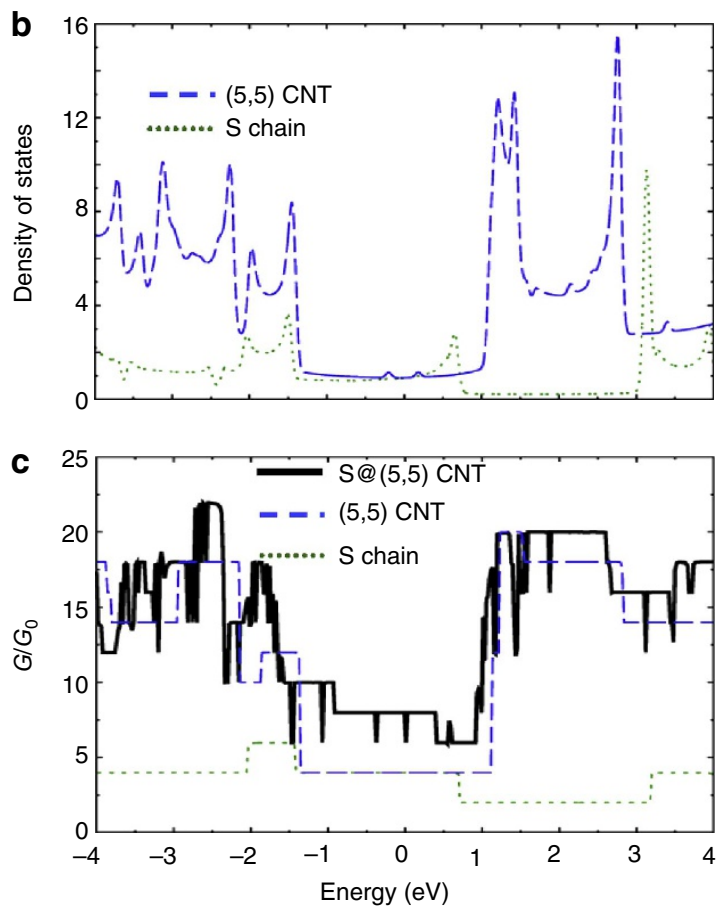

Figure 6 | Electronic properties of a sulphur chain inside a CNT. (a) Side and end-on view of a sulphur chain encapsulated inside a $(5,5)$ SWCNT. (b) Electronic density of states of a $(5,5)$ SWCNT and an isolated sulphur chain. (c) Ballistic quantum conductance of a sulphur chain encapsulated inside a $(5,5)$ SWCNT along with that of an isolated SWCNT and an isolated sulphur chain. $E=0$ denotes the Fermi level in $\mathbf{b}$ and the carrier injection energy in a neutral, unbiased system in c. $G_{0}$ is the conductance quantum. 
calculations of isolated sulphur chains and sulphur chains contained inside the inner core of $(5,5)$ and $(8,0)$ CNTs (see Supplementary Figs S12-S15). Our results for the quantum conductance of a sulphur chain encapsulated inside a $(5,5)$ CNT are presented in Fig. 6. The sulphur chain and the CNT are both metallic, because their electronic densities of states are nonzero at the Fermi level (see Fig. 6b). As the density of states of S@CNT in Fig. $6 \mathrm{~b}$ is close to the superposition of that of a neutral nanotube and a sulphur chain, we conclude that there is no significant bonding or charge transfer between the chain and the nanotube. Our Mulliken population analysis indicates a negligible charge transfer of only $\sim 0.1$ electrons/sulphur atom between sulphur and the surrounding CNT (see Supplementary Note 6). The quantum conductance of the S@SWCNT system, as well as its components, calculated using the scattering geometry illustrated in Fig. 6a, is shown in Fig. 6c. As mentioned above, the electronic interaction between the encapsulated sulphur chain and the enclosing nanotube is very weak. Then, the total conductance of the S@SWCNT system should be close to the superposition of the quantum conductances of the isolated chain and the isolated nanotube. These quantum conductances are plotted in Fig. 6c, together with the conductance of the S@SWCNT. In each case, as indicated in Fig. 6a, we used the same system to describe the leads and the scattering region. With $G=8 G_{0}$, we find the quantum conductance of the unbiased and neutral S@SWCNT system to be twice as high as the M-SWCNT. Even at other injection energies, the conductance of the S@SWCNT system lies very close to the superposition of the isolated sulphur chain and the SWCNT, with the exception of dips in the conductance curve that are characteristic of the small sulphur-SWCNT interaction. Thus, our calculations provide solid support for the conclusion that sulphur chains do not modify the electronic conductance of CNTs, but rather introduce new conduction channels in the S@CNT system.

\section{Discussion}

We have experimentally identified the presence of conducting 1D zigzag and linear sulphur chains, which self-assemble in the narrow cylindrical cavity inside SWCNTs and DWCNTs. Our $\mathrm{XRD}$ analysis reveals the long-range order and large domain size of the 1D sulphur chains inside CNTs at $300 \mathrm{~K}$, resulting from the strong covalent bond in the 1D sulphur chains. Because of the covalent bonding character, we found high thermal stability of the $1 \mathrm{D}$ sulphur chains inside CNTs. The $1 \mathrm{D}$ sulphur chains retain those 1D structures up to $800 \mathrm{~K}$ of which temperature is much higher than boiling point of the bulk $S_{8}$. Those results, as also supported by negligible charge transfer between 1D sulphur chains and CNTs, lead us to believe that the 1D sulphur chains act as a previously not observed conducting polymer consisting only of elemental sulphur, thus improving the electrical conductivity of CNTs.

The identification of a metallic phase of sulphur at ambient pressures is highly noteworthy, because bulk sulphur requires very high pressures $>90 \mathrm{GPa}$ to become metallic. We expect that the present results for CNT enclosures may be realized also in other nanoporous systems, including covalent organic frameworks ${ }^{44}$ and zeolites ${ }^{45}$, thus opening new avenues to study the chemistry of sulphur at the nanoscale. Since the electronic structure changes under steric constraints are not limited to sulphur, we foresee a new branch of chemistry evolving in the area of artificial stabilization of exotic structures inside nanoporous systems.

\section{Methods}

Synthesis of 1D chains of sulphur. Reagents were obtained from commercial suppliers and used without further purification. The SWCNT sample produced by the arc-discharged method was provided by Hanwha Nanotech, and the DWCNT sample was purchased from Toray Industries, Inc. The sorted sample of M-SWCNTs (99\%) was purchased from NanoIntegris Inc. and used after annealing treatment at $773 \mathrm{~K}$ under $1 \mathrm{~Pa}$ for $1 \mathrm{~h}$. High-purity (99.9999\%), bright-yellow crystal of sulphur was used for avoiding contaminants. To encapsulate sulphur in the CNTs efficiently, the terminating caps on the SWCNT and DWCNT samples were first removed by oxidation treatment at $723 \mathrm{~K}$ under dry air $\left(100 \mathrm{ml} \mathrm{min}^{-1}\right)$ for $1 \mathrm{~h}$. The open-ended CNT samples and the crystalline sulphur were sealed in a forked glass tube in vacuo $(<1 \mathrm{~Pa})$ and subsequently kept at $873 \mathrm{~K}$ for $48 \mathrm{~h}$. The asprepared sample was then washed with carbon disulphide to dissolve excess sulphur attached to the outside of the CNTs. Finally, S@CNT sheets (buckypaper) were obtained after filtration of the S@CNT-carbon disulphide solution and subsequent drying at $383 \mathrm{~K}$ in vacuo $(<1 \mathrm{~Pa})$.

Analytical techniques. HRTEM observations were carried out with a double Cs-corrected (CEOS GmbH) HRTEM (JEM-2100F; JEOL) operated at $80 \mathrm{kV}$. XRD profiles were measured using the synchrotron X-ray source at SPring-8

$(\lambda=0.08003 \mathrm{~nm})$. The samples were sealed in a glass capillary in vacuo $(<1 \mathrm{~Pa})$. Temperature was controlled using an $\mathrm{N}_{2}$ gas injection gun. XPS measurements were performed using a monochromatized AlK $\alpha$ X-ray source (AXIS-ULTRA DLD; Shimazu). TG measurements were carried out under $\mathrm{He} / \mathrm{O}_{2}$ (8:2) gas flow in the temperature range $300-1,200 \mathrm{~K}$ at a scan rate of $3 \mathrm{~K} \mathrm{~min}^{-1}$ (Thermo-Mass; Rigaku Corporation). The flow rate was kept at $300 \mathrm{ml} \mathrm{min}^{-1}$. Elemental mapping analyses were performed by energy-dispersive spectroscopy. The spectroscope (JED-2300T; JEOL) was coupled to a scanning transmission electron microscope operated at $120 \mathrm{keV}$. Raman spectra were obtained with 532 and $785 \mathrm{~nm}$ excitations, using a single-monochromator micro-Raman spectrometer in back scattering configuration (inVia Raman Microscope; Renishaw). Direct-current electric-resistance measurements were performed by the four-probe method, using a physical properties measurement system (model 6000; Quantum Design) in the temperature range from room temperature to $2 \mathrm{~K}$. Transport measurements were performed on the S@CNT sheet (buckypaper).

Computational methods. Our calculations of the equilibrium structure, stability and electronic properties of sulphur chains inside SWCNTs have been performed using $a b$ initio density functional theory, as implemented in the SIESTA code ${ }^{46}$. We used the spin-polarized Ceperley-Alder ${ }^{47}$ exchange-correlation functional as parameterized by Perdew and Zunger ${ }^{48}$, norm-conserving Troullier-Martins pseudopotentials ${ }^{49}$ and a double- $\zeta$ basis including polarization orbitals. We used periodic boundary conditions to represent arrays of aligned, but well separated, SWCNTs containing sulphur. The Brillouin zone of the isolated 1D chain and nanotube structures was sampled by at least the equivalent of $16 k$-points. We used a mesh cutoff energy of 180 Ry to determine the self-consistent charge density, which provided us with a precision in total energy of $<7 \mathrm{meV}$ per atom. Quantum transport calculations were performed using the TRANSIESTA $\operatorname{code}^{50}$, based on the non-equilibrium Green's function approach.

\section{References}

1. Steudel, R. \& Eckert, B. Solid sulfur allotropes. Top. Curr. Chem. 230, 1-79 (2003).

2. Amaya, K. et al. Observation of pressure-induced superconductivity in the megabar region. J. Phys.: Condens. Matter 10, 11179 (1998).

3. Akahama, Y., Kobayashi, M. \& Kawamura, H. Pressure-induced structural phase transition in sulfur at $83 \mathrm{GPa}$. Phys. Rev. B 48, 6862-6864 (1993).

4. Luo, H., Desgreniers, S., Vohra, Y. K. \& Ruoff, A. L. High-pressure optical studies on sulfur to $121 \mathrm{GPa}$ : Optical evidence for metallization. Phys. Rev. Lett 67, 2998-3001 (1991).

5. Springborg, M. \& Jones, R. O. Energy surfaces of polymeric sulfur: structure and electronic properties. Phys. Rev. Lett. 57, 1145-1148 (1986).

6. Springborg, M. \& Jones, R. O. Sulfur and selenium helices: structure and electronic properties. J. Chem. Phys. 88, 2652-2658 (1988).

7. Okano, S. \& Tománek, D. Effect of electron and hole doping on the structure of C, Si, and S nanowires. Phys. Rev. B 75, 195409 (2007).

8. Peierls, R. E. Quantum Theory of Solids (Oxford University Press, New York, 1955).

9. Su, W. P., Schrieffer, J. R. \& Heeger, A. J. Solitons in polyacetylene. Phys. Rev. Lett. 42, 1698-1701 (1979)

10. Okada, S., Saito, S. \& Oshiyama, A. Energetics and electronic structures of encapsulated $\mathrm{C}_{60}$ in a carbon nanotube. Phys. Rev. Lett. 86, 3835-3838 (2001).

11. Giménez-López, M. d. C. et al. Encapsulation of single-molecule magnets in carbon nanotubes. Nat. Commun. 2, 407 (2011).

12. Chuvilin, A. et al. Self-assembly of a sulphur-terminated graphene nanoribbon within a single-walled carbon nanotube. Nat. Mater. 10, 687-692 (2011).

13. Urita, K. et al. Confinement in carbon nanospace-induced production of KI nanocrystals of high-pressure phase. J. Am. Chem. Soc. 133, 10344-10347 (2011).

14. Maniwa, Y. et al. Ordered water inside carbon nanotubes: formation of pentagonal to octagonal ice-nanotubes. Chem. Phys. Lett. 401, 534-538 (2005). 
15. Sloan, J., Kirkland, A. I., Hutchison, J. L. \& Green, M. L. H. Integral atomic layer architectures of $1 \mathrm{D}$ crystals inserted into single walled carbon nanotubes. Chem. Commun. 1319-1332 (2002).

16. Fan, X. et al. Atomic arrangement of iodine atoms inside single-walled carbon nanotubes. Phys. Rev. Lett. 84, 4621-4624 (2000).

17. Kitaura, R., Imazu, N., Kobayashi, K. \& Shinohara, H. Fabrication of metal nanowires in carbon nanotubes via versatile nano-template reaction. Nano Lett. 8, 693-699 (2008).

18. Smith, B. W., Monthioux, M. \& Luzzi, D. E. Encapsulated $\mathrm{C}_{60}$ in carbon nanotubes. Nature 396, 323-324 (1998).

19. Chernysheva, M. V. et al. The electronic properties of SWNTs intercalated by electron acceptors. Physica E 40, 2283-2288 (2008).

20. Saito, R., Dresselhaus, G. \& Dresselhaus, M. S. Physical Properties of Carbon Nanotubes (Imperial College Press, 1998).

21. Mintmire, J. W., Dunlap, B. I. \& White, C. T. Are fullerene tubules metallic? Phys. Rev. Lett. 68, 631-634 (1992).

22. Kataura, H. et al. High-yield fullerene encapsulation in single-wall carbon nanotubes. Synth. Met. 121, 1195-1196 (2001).

23. Chen, C.-E., Schlesinger, Y. \& Heeger, A. J. X-ray scattering by onedimensional chains: powder diffraction. Phys. Rev. B 25, 2472-2476 (1982).

24. Mohan, M. M. Structure factor of a single linear chain of finite size. Phys. Rev. $B$ 34, 8915-8921 (1986).

25. Abe, M. et al. Structural transformation from single-wall to double-wall carbon nanotube bundles. Phys. Rev. B 68, 041405(R) (2003).

26. Knacke, O., Kubaschewski, O. \& Hesselmann, K. Thermochemical Properties of Inorganic Substances 2nd edn (Springer Verlag, 1991).

27. Crapanzano, L. et al. Alternating sequence of ring and chain structures in sulphur at high pressure and temperature. Nat. Mater. 4, 550-552 (2005).

28. Degtyareva, O. et al. Novel chain structures in group VI elements. Nat. Mater. 4, 152-155 (2005)

29. Fujihisa, H. et al. Spiral chain structure of high pressure selenium-II' and sulfur-II from powder X-ray diffraction. Phys. Rev. B 70, 134106 (2004).

30. Eckert, B. \& Steudel, R. Molecular spectra of sulfur molecules and solid sulfur allotropes. Top. Curr. Chem. 231, 31-98 (2003).

31. Pauling, L. The Nature of The Chemical Bonding and The Structure of Molecules and Crystals: An Introduction to Modern Structural Chemistry 3rd edn (Cornell University Press, Ithaca, NY, 1938).

32. Jorio, A. et al. Characterizing carbon nanotube samples with resonance Raman scattering. N. J. Phys. 5, 139.1-139.17 (2003).

33. Brown, S. D. M. et al. Origin of the Breit-Wigner-Fano lineshape of the tangential $G$-band feature of metallic carbon nanotubes. Phys. Rev. B 63, 155414 (2001).

34. Bandow, S. et al. Evidence for anomalously small charge transfer in doped single-wall carbon nanohorn aggregates with Li, K and Br. Appl. Phys. A 71, 561-564 (2000)

35. Eklund, P. C. \& Subbaswamy, K. R. Analysis of Breit-Wigner line shapes in the Raman spectra of graphite intercalation compounds. Phys. Rev. B 20, 5157-5161 (1979).

36. Kataura, H. et al. Optical properties of single-wall carbon nanotubes. Synth. Met. 103, 2555-2558 (1999).

37. Maciel, I. O. et al. Electron and phonon renormalization near charged defects in carbon nanotubes. Nat. Mater. 7, 878-883 (2008).

38. Green, A. A. \& Hersam, M. C. Colored semitransparent conductive coatings consisting of monodisperse metallic single-walled carbon nanotubes. Nano Lett. 8, 1417-1422 (2008).

39. Arnold, M. S., Stupp, S. I. \& Hersam, M. C. Enrichment of single-walled carbon nanotubes by diameter in density gradients. Nano Lett. 5, 713-718 (2005).

40. Mott, N. Conduction in Non-Crystalline Materials (Oxford University Press, 1987).
41. Yanagi, K. et al. Transport mechanisms in metallic and semiconducting singlewall carbon nanotube networks. ACS Nano 4, 4027-4032 (2010).

42. Fujisawa, K. et al. Chirality-dependent transport in double-walled carbon nanotube assemblies: The role of inner tubes. ACS Nano 5, 7547-7554 (2011)

43. Hirahara, K. et al. One-dimensional metallofullerene crystal generated inside single-walled carbon nanotubes. Phys. Rev. Lett. 85, 5384-5387 (2000).

44. Côté, A. P. et al. Porous, crystalline, covalent organic frameworks. Science 310, 1166-1170 (2005).

45. Herron, N. \& Corbin, D. R. Inclusion Chemistry with Zeolites: Nanoscale Materials by Design (Kluwer Academic Publishers, 1995).

46. Artacho, E. et al. The SIESTA method; developments and applicability. J. Phys. Cond. Matter 20, 064208 (2008).

47. Ceperley, D. M. \& Alder, B. J. Ground state of the electron gas by a stochastic method. Phys. Rev. Lett. 45, 566-569 (1980).

48. Perdew, J. P. \& Zunger, A. Self-interaction correction to density-functional approximations for many-electron systems. Phys. Rev. B 23, 5048-5079 (1981).

49. Troullier, N. \& Martins, J. L. Efficient pseudopotentials for plane-wave calculations. Phys. Rev. B 43, 1993-2006 (1991).

50. Brandbyge, M. et al. Density-functional method for nonequilibrium electron transport. Phys. Rev. B 65, 165401 (2002).

\section{Acknowledgements}

T.F., H.M., M.T., M.E. and K.K. were supported by Exotic Nanocarbons, Japan Regional Innovation Strategy Program by the Excellence, JST. The synchrotron radiation experiments were performed at the BL02B2 of SPring- 8 with the approval of the Japan Synchrotron Radiation Research Institute (JASRI) (Proposal No. $2011 \mathrm{~B} 1559$ and 2012B1064). A.M.-G was in part supported by The New Energy and Industrial Technology Development Organization (NEDO) for electrical conductivity analysis. T.F. and T.H. were supported by a Grant-in-Aid for Young Scientists (B) (No. 25820331) and a Grant-in-Aid for Scientific Research (C) (No. 22510112) of the Japan Society for the Promotion of Science, respectively. D.T. and Z.Z. were partially supported by the National Science Foundation Cooperative Agreement no. EEC-0832785, titled 'NSEC: Center for High-rate Nanomanufacturing'. Computational resources have been provided by the Michigan State University High Performance Computing Center. T.F. thanks H. Terrones for fruitful discussions.

\section{Author contributions}

T.F. conceived the project. T.F., M.T. and D.T. prepared the manuscript. T.F., A.M.-G., H.M., K.U., and T.H. carried out the experiments. R.F. supported interpretation of the XRD data. Z.Z. performed DFT and transport calculations. S.Y.H. and Y.C.C. produced the SWCNT sample. M.T., M.E., D.T. and K.K. supervised the research.

\section{Additional information}

Supplementary information accompanies this paper at www.nature.com/ naturecommunications/

Competing financial interests: The authors declare no competing financial interests.

Reprint and permissions information is available at http://npg.nature.com/ reprintsandpermissions/

How to cite this article: Fujimori, T. et al. Conducting linear chains of sulphur inside carbon nanotubes. Nat. Commun. 4:2162 doi: 10.1038/ncomms3162 (2013).

This article is licensed under a Creative Commons Attribution 3.0 Unported Licence. To view a copy of this licence visit http:// creativecommons.org/licenses/by/3.0/ 